\title{
Affording Enjoyment in VR Games: Possibilities, Pitfalls, and Perfection
}

\author{
PENNY SWEETSER and ZANE ROGALEWICZ, The Australian National University, Australia
}

In this paper, we present research that aims to understand affordances and inhibitors of enjoyment in virtual reality (VR) video games. We apply the GameFlow model to analyse VR and non-VR versions of the same games to identify differences in enjoyment in VR games. Our approach involves conducting qualitative analysis on video game reviews, using GameFlow as a theoretical foundation. We report on our analysis of the games Superhot and Skyrim. We find that affordances are largely consistent between VR and non-VR versions of the same games, with a few key differences related to Feedback, Control, Player Skills, and Immersion. We conclude that GameFlow is applicable to VR games, with the addition of a Comfort element to describe player comfort while playing.

CCS Concepts: • Human-centered computing $\rightarrow$ Heuristic evaluations; Virtual reality; $\bullet$ Software and its engineering $\rightarrow$ Interactive games.

Additional Key Words and Phrases: virtual reality, videogames, enjoyment, player experience, GameFlow

\section{ACM Reference Format:}

Penny Sweetser and Zane Rogalewicz. 2020. Affording Enjoyment in VR Games: Possibilities, Pitfalls, and Perfection. In 32ND AUSTRALIAN CONFERENCE ON HUMAN-COMPUTER INTERACTION (OzCHI '20), December 2-4, 2020, Sydney, NSW, Australia. ACM, New York, NY, USA, 15 pages. https://doi.org/10.1145/3441000.3441050

\section{INTRODUCTION}

GameFlow [64] is a model of player enjoyment in video games, which includes 38 criteria within eight elements. GameFlow was derived from games user experience heuristics and mapped to the elements of Csikszentmihalyi's [12] concept of flow. The GameFlow model has seen extensive application in the design and evaluation of games and game-like experiences, including extended reality (XR - e.g., virtual, augmented, and mixed reality). However, previous applications of the GameFlow model to XR have focused on evaluating a particular game or experience, rather than seeking to understand enjoyment in these experiences more broadly.

In the study reported in this paper, we aim to apply the GameFlow model to understand how enjoyment can be supported and enhanced in VR games. We also seek to identify inhibitors of enjoyment in VR games. Our ongoing research also aims to test the GameFlow model's applicability to different games and platforms [56-63]. Our approach for the research reported in this paper involves carrying out qualitative analysis of professional game reviews of a selection of VR games, as well as their non-VR counterparts, using the GameFlow model as a theoretical framework. In this paper, we explore previous applications of GameFlow to XR games and experiences, define our methodology and approach, and report on the results of our qualitative analysis of professional game reviews. We conclude that affordances of enjoyment are similar across VR and non-VR games, with the exception of opportunities and issues related to physical involvement. We also propose the addition of a Comfort element to the GameFlow model when considering VR games.

Permission to make digital or hard copies of all or part of this work for personal or classroom use is granted without fee provided that copies are not made or distributed for profit or commercial advantage and that copies bear this notice and the full citation on the first page. Copyrights for components of this work owned by others than the author(s) must be honored. Abstracting with credit is permitted. To copy otherwise, or republish, to post on servers or to redistribute to lists, requires prior specific permission and/or a fee. Request permissions from permissions@acm.org.

(c) 2020 Copyright held by the owner/author(s). Publication rights licensed to ACM.

Manuscript submitted to ACM 
Table 1. GameFlow elements for an enjoyable game experience [64].

\begin{tabular}{|c|c|}
\hline Element & Description \\
\hline Concentration & $\begin{array}{l}\text { The game should require concentration and the player should be able to } \\
\text { concentrate on the game. }\end{array}$ \\
\hline Challenge & The game should be sufficiently challenging and match the player's skill level. \\
\hline Player Skills & The game must support player skill development and mastery \\
\hline Control & Players should feel a sense of control over their actions in the game. \\
\hline Clear Goals & The game should provide the player with clear goals at appropriate times. \\
\hline Feedback & Players must receive feedback at appropriate times. \\
\hline Immersion & Players should experience deep but effortless involvement in the game. \\
\hline Social Interaction & The game should support and create opportunities for social interaction. \\
\hline
\end{tabular}

\section{GAMEFLOW IN EXTENDED REALITY}

The GameFlow model, which consists of eight elements of player enjoyment in games (see Table 1), has been extensively applied to the design and evaluation of games and experiences for a variety of purposes, audiences, platforms, and domains. Among these applications are various XR games and experiences (see Table 2), including designing VR games and simulations [2, 15, 68], evaluating VR experiences [1, 15, 22], analysing and evaluating Augmented Reality (AR) experiences [35, 40, 72, 73], designing AR games [23], and evaluating Mixed Reality (MR) games [25, 65]. XR experiences that the GameFlow model has been applied to include applications for overcoming phobias [1], exercise and sports games and simulations [2, 15, 23, 73], audio-based games [68, 72], artworks [22], and maps [35]. Although a number of studies in VR, AR, and MR have applied the GameFlow model for the purposes of design and/or evaluation, this research has not sought to understand more broadly what constitutes an enjoyable XR experience. In the research reported in this paper, we use the GameFlow model to both evaluate VR games and to build up an understanding of enjoyment in VR games.

\section{METHOD}

We conducted a qualitative analysis on sets of professional game reviews, using deductive Thematic Analysis [11] with GameFlow as a theoretical framework. Game reviews are appropriate for analysing as data to capture the play experience as they "in some sense, convey the experience of playing video-games" [10]. Previous research has involved analysing game reviews to characterise good and bad games [6], to develop models of the player experience [10], and to compile detailed GameFlow heuristics [60,62]. This approach offered detailed insights into how the GameFlow criteria are afforded in VR versus non-VR games, as well as identifying opportunities for designing VR games to enhance enjoyment.

We selected two games to analyse that have both VR and non-VR versions, Superhot and Skyrim. We analysed 10 professional game reviews for each version of each game, as in previous work $[60,62]$. Reviews were selected from more popular, reputable critic websites, with longer, more detailed reviews preferred. Table 3 presents the full list of reviews analysed. Table 4 summarises the details of the selected games, including release year, genre, and aggregate review scores according to Metacritic [30]. 
Table 2. Applications of GameFlow to Virtual Reality (VR), Augmented Reality (AR), and Mixed Reality (MR).

\begin{tabular}{|c|c|}
\hline VR Applications & Description \\
\hline Virtual Showdown [68] & $\begin{array}{l}\text { GameFlow criteria used to improve the design of a VR game with audio as } \\
\text { primary cues for youth with visual impairment. }\end{array}$ \\
\hline La Camera Insabbiata [22] & Questionnaire based on GameFlow used to evaluate award-winning VR artwork. \\
\hline VR sports training [2] & GameFlow followed to design an Olympic pistol shooting training simulation. \\
\hline VR phobia apps [1] & $\begin{array}{l}\text { Questionnaire based on GameFlow used to evaluate player enjoyment in two } \\
\text { VR game prototypes for overcoming fear of heights. }\end{array}$ \\
\hline Astrojumper [15] & GameFlow used in the design and evaluation of a VR exercise game. \\
\hline VR exergame [3] & GameFlow used in the design of a VR exercise game. \\
\hline Visuo-Haptic Game [39] & Gamification sub-components inspired by GameFlow. \\
\hline AR Applications & Description \\
\hline Exertion Interface [73] & $\begin{array}{l}\text { Questionnaire based on GameFlow used to evaluate sports exercises in AR, } \\
\text { with and without gamification. }\end{array}$ \\
\hline Mobile AR games [40] & GameFlow elements used to analyse selected AR games. \\
\hline Quantum Games [72] & $\begin{array}{l}\text { GameFlow used to analyse probabilistic physical AR games with a non-visual } \\
\text { (audio only) imaginary ball. }\end{array}$ \\
\hline The Skeleton Chase [23] & GameFlow used as a design standard for AR game for increasing physical activity. \\
\hline MapLens [35] & $\begin{array}{l}\text { Questionnaire based on GameFlow used to measure user experience in an AR } \\
\text { map for mobile devices. }\end{array}$ \\
\hline MR Applications & Description \\
\hline Age Invaders [25] & GameFlow was used to evaluate a social-physical inter-generational MR game. \\
\hline Metazoa Ludens [65] & $\begin{array}{l}\text { Questionnaire based on GameFlow used to evaluate a system that enables } \\
\text { humans to play games with small animals in a MR environment. }\end{array}$ \\
\hline Tainted [42] & Questionnaire based on GameFlow used to evaluate an olfactory game. \\
\hline Exercube [28] & Questionnaire based on GameFlow used to assess an immersive game. \\
\hline MIRA Rehab software [29] & Interview guided by GameFlow used to evaluate exergame for older people. \\
\hline Plunder Planet [27] & Questionnaire based on GameFlow used to evaluate adaptive fitness game. \\
\hline Eyes-Free Yoga [43] & Interview based on GameFlow used to evaluate low vision exergame. \\
\hline
\end{tabular}

\subsection{Selected Games}

SuperHot is a first-person shooter (FPS) game in which the player is tasked with defeating all the enemies in a level. In the game, time flows when the player moves and at a speed relative to the player's movements. If the player is standing still, then all enemies and projectiles also freeze in place, giving the player time to consider their next actions.

Skyrim is a role-playing game where the player controls a character and explores an expansive open world. In the game, the player has characters to interact with, enemies to defeat, stories to uncover, and abilities to learn. The player has the freedom to choose their next objective and is never locked to a linear path. 
Table 3. Professional game reviews used as data for each selected game.

\begin{tabular}{|c|c|}
\hline Game & Review sources \\
\hline Superhot & $\begin{array}{l}\text { Attack of the Fanboy [18], Game Informer [44], Hardcore Gamer [71], } \\
\text { GameCritics.com [55], Destructoid [13], Gamespot [8], IGN [38], } \\
\text { Metro News [31], PC Gamer [47], The Escapist [46]. }\end{array}$ \\
\hline Superhot VR & $\begin{array}{l}\text { Attack of the Fanboy [20], Dual Shockers [34], GameCritics.com [69], } \\
\text { Game Informer [17], IGN [53], Gaming Nexus [9], Metro News [33], } \\
\text { PlayStation Universe [24], SSVAR [50], Video Chums [26]. }\end{array}$ \\
\hline Skyrim & $\begin{array}{l}\text { Destructoid [54], Eurogamer.net [4], GameChronicles [7], } \\
\text { GameInformer [45], GameSpy [36], Giant Bomb [49], IGN [37], } \\
\text { PC Gamer [16], Video Gamer [5], Wired [48]. }\end{array}$ \\
\hline Skyrim VR & $\begin{array}{l}\text { Attack of the Fanboy [19], CGMagazine [41], Critical Hit [66], } \\
\text { Gaming Age [14], Metro News [32], Hardcore Gamer [70], IGN [52], } \\
\text { Impulse Gamer [67], Game Informer [21], SSVAR [51]. }\end{array}$ \\
\hline
\end{tabular}

Table 4. Evaluated games with year released, game genre, and aggregate review score according to Metacritic [30].

\begin{tabular}{lllrr}
\hline Game & Year & Genre & Critic Score & User Score \\
\hline Superhot & 2016 & First-person shooter & $82 \%(93$ reviews $)$ & $7.5(513$ reviews $)$ \\
Superhot VR & 2016 & First-person shooter & $83 \%(19$ reviews $)$ & $7.5(44$ reviews $)$ \\
Skyrim & 2011 & Role-playing & $94 \%(32$ reviews $)$ & $8.2(10735$ reviews $)$ \\
Skyrim VR & 2018 & Role-playing & $81 \%(11$ reviews $)$ & $6.9(57$ reviews $)$ \\
\hline
\end{tabular}

\section{CODING OF GAME REVIEWS}

One rater coded the reviews against the GameFlow elements and criteria, with a second rater independently coding $5 \%$ of the reviews ( 2 complete reviews). Following the coding process, the two raters discussed the discrepancies in their coding and the coding was revisited by the first rater. Reviews were coded against the 8 GameFlow elements and the 38 criteria. Any statements in the reviews that did not fit the GameFlow elements and criteria were coded into new categories. The coding and analysis were performed using NVivo 12.

Table 5 shows a summary of the qualitative coding of the professional game reviews, including total number of positive, neutral, and negative comments coded into each GameFlow element for each game. Social Interaction is not included as all games selected are single player games. An additional theme that emerged from the data, Comfort, is also presented. One point of interest is that there were substantially more comments coded into Concentration for the non-VR games (168) than the VR games (38). It is also notable that there were more negative comments for VR games than the non-VR games for Player Skills (31:6), Feedback (10:4), and Immersion (24:12). For Control, the VR games had a similar number of positive (42) and negative (38) comments.

Comfort was a theme that emerged that was not covered by the existing GameFlow elements. Comfort was only present in VR game reviews. More comments were coded into Comfort for Skyrim VR (28) than for Superhot VR (15). There were also more negative comments (23) for Comfort than positive (18). 
Table 5. Total number of positive (+), neutral (/), and negative (-) comments for each game by GameFlow element.

\begin{tabular}{llrrr}
\hline Element & Game & + & $/$ & - \\
\hline Concentration & Skyrim & 107 & 6 & 9 \\
& Skyrim VR & 15 & 1 & 5 \\
& Superhot & 28 & 0 & 8 \\
& Superhot VR & 10 & 0 & 6 \\
\hline Challenge & Skyrim & 11 & 2 & 3 \\
& Skyrim VR & 1 & 1 & 7 \\
& Superhot & 24 & 0 & 4 \\
& Superhot VR & 12 & 0 & 3 \\
\hline Player Skills & Skyrim & 34 & 0 & 1 \\
& Skyrim VR & 23 & 0 & 21 \\
& Superhot & 48 & 3 & 5 \\
& Superhot VR & 43 & 0 & 10 \\
\hline Control & Skyrim & 76 & 1 & 9 \\
& Skyrim VR & 28 & 0 & 28 \\
& Superhot & 23 & 0 & 4 \\
& Superhot VR & 14 & 1 & 10 \\
\hline Clear Goals & Skyrim & 19 & 1 & 2 \\
& Skyrim VR & 3 & 0 & 0 \\
& Superhot & 6 & 0 & 0 \\
& Superhot VR & 4 & 0 & 0 \\
\hline Feedback & Skyrim & 10 & 0 & 2 \\
& Skyrim VR & 11 & 0 & 6 \\
& Superhot & 2 & 0 & 2 \\
& Superhot VR & 3 & 0 & 4 \\
\hline & Skyrim & 59 & 0 & 5 \\
& Skyrim VR & 43 & 0 & 17 \\
& Superhot & 18 & 1 & 7 \\
& Superhot VR & 33 & 0 & 7 \\
\hline & Skyrim & 0 & 0 & 0 \\
& Skyrim VR & 11 & 0 & 17 \\
& Superhot & 0 & 0 & 0 \\
& 7 & 2 & 6 \\
\hline \multirow{7}{*}{ Comfort } & & & \\
& &
\end{tabular}

\section{ANALYSIS OF GAME REVIEWS}

In this section, we report the results of the thematic analysis of the professional game reviews. We present a summary of how each GameFlow element is afforded by the selected games and key points of differentiation between the VR and non-VR versions of each game. Social Interaction is not included as all games selected are single player games. An additional theme that emerged from the data, Comfort, is also presented. 


\subsection{Concentration}

There were no notable differences in affordances for Concentration between the VR versus non-VR games. In Skyrim, Concentration is afforded in the form of the amount and variety of content, visuals, non-player character interactions, quality of the game, and the quests. In Superhot, Concentration is afforded via the uniqueness of mechanics, the intensity of gameplay, the narrative, the level design, and the presentation of the game. Reviewers noted that Superhot's narrative interrupts gameplay throughout the game, which received mixed reviews. The VR release of the game removed most of the original narrative with reviewers considering what was left to be an unimportant distraction (e.g., "the plot of Superhot VR is thin enough that it often feels quite superfluous" [24], "it would be great to see more narrative woven into the gameplay, or maybe just eliminate it all together - right now what's there feels like a novel distraction" [17]).

\subsection{Challenge}

There were no notable differences in affordances for Challenge between the VR versus non-VR games. In Superhot, Challenge is afforded by the number of active enemies, the weapons they hold, their distance from the player, the environment in which the level occurs, the weapons available to the player, and how the player uses the time flow ability 1. In Skyrim, Challenge is afforded by the level difference between the enemy and player, the abilities enemies possess, the enemy AI, the environment in which the encounter occurs, and the options the player has for an engagement. The Skyrim VR reviews mentioned that its implementation breaks some of the mechanics (e.g., "you'll get steered off to the side if you try to aim while your horse is moving" [51]) of the game and that there is a lack of new content for returning players.

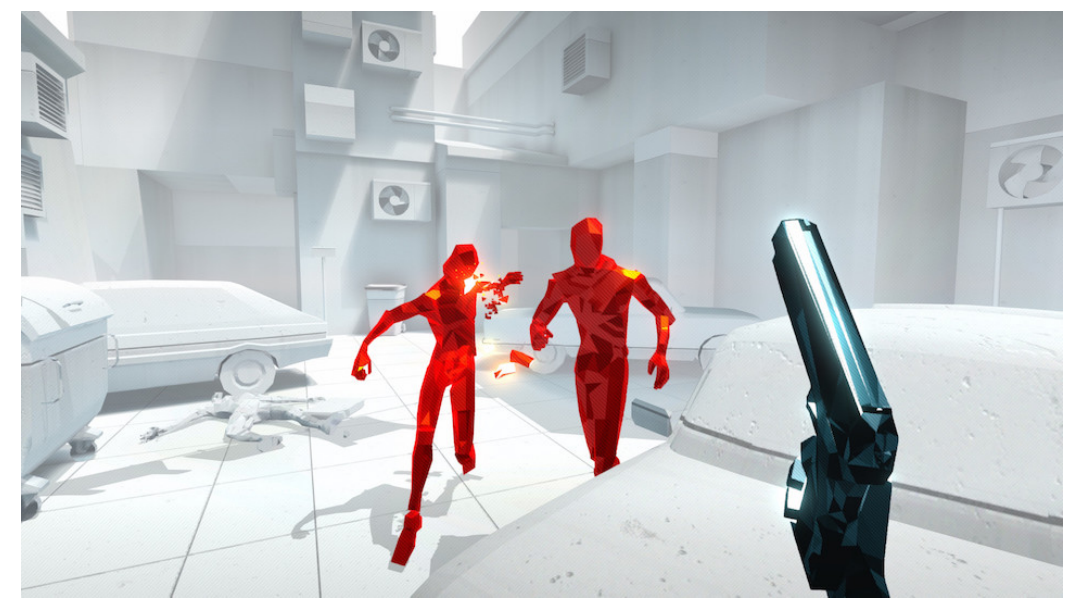

Fig. 1. Superhot affords Challenge via enemies, weapons, distance, and environment.

\subsection{Player Skills}

The key differences in affordances for Player Skills between the VR and non-VR games related to the Player Skills criteria:

- Players should be rewarded appropriately for their effort and skill development.

- Game interfaces and mechanics should be easy to learn and use. 
In Skyrim, the Player Skills element is afforded through combat, skill progression, story progression, menu design, and the introductory sequence. Skyrim VR reviews mentioned that the control system makes learning mechanics more enjoyable (e.g., "it turns out the melee attacks from horseback are really fun" [51]) and that the VR experience rewards players with satisfaction for mastery (e.g., "feels good to actually hold your shield up to block attacks and bash enemies" [51], "no more satisfying feeling than striking an enemy with your sword whilst casually blasting force lighting with your free hand" [67], "seeing a stream of fire shoot from your hands is an empowering feeling" [52]). Reviews also noted that the combat system is intuitive as the player is physically performing the actions (e.g., "using a sword and shield, you grip the Move controllers as if you were holding the items, raising your left hand to block and swinging your right hand is if you were holding a sword" [21]). However, archery combat was perceived to not translate well to VR (e.g., "aim is really only done with your bow hand, which feels less intuitive and natural" [51], "I did find using the bow to be incredibly difficult during my first few hours of exploring" [67], see Figure 2). The user interface elements were also highlighted as not translating well due to their positioning on the VR display (e.g., "one thing I had an issue with was the compass and looking down" [70]).

In Superhot, the Player Skills element is afforded through combat mechanics, game progression, and time mechanics. Superhot VR reviews mentioned that the throwing mechanic did not translate well to VR (e.g., "I'm sure the developers are loathe to include a simplified point-and-shoot throwing mode since it would break the immersion that the game is built around, but right now the throwing is so frustrating it made me want to quit more than once" [69]). The VR version does not have the playback feature (i.e., plays back an action sequence), which is rewarding for the player. However, physically performing the super-human actions in the VR version is rewarding in itself ("watching trails of bullets whip by your head as you carefully duck and dodge out of harm's way is exhilarating" [26], "more than anything else, Superhot VR makes you feel like a legitimate action movie hero" [34], "in Superhot VR, because you are physically performing these acts yourself, the feeling of satisfaction is that much greater" [24]). Physically performing the actions also makes the combat system more intuitive (e.g., "picking up weapons with the grip handles of the Vive controllers makes a lot of sense" [20]). Reviewers also noted that the hit detection does not always line up with player expectations (e.g., "every once in a while a bullet will come close to your head and still kill you even though it might have seemed like it shouldn't have" [17]) and the progression button is unintuitive in its placement within the game world.

\subsection{Control}

The key differences in affordances for Controls between the VR and non-VR games related to the Control criteria:

- Players should feel a sense of control over their characters or units and their movements and interactions in the game world.

- Players should not be able to make errors that are detrimental to the game and should be supported in recovering from errors.

In Skyrim, Control is afforded by the open world, the content available, the character customisation, and the combat. Reviewers described how they have more control over how they engage in combat scenarios in Skyrim VR (e.g., "using the Moves also opens up a whole new dimension in terms of combat, giving players the opportunity to simultaneously attack in two different directions" [67]) and how the VR perspective increases feelings of connectedness to the world (e.g., "I felt more connected to the world than through a flat screen, I didn't feel the urge to try randomly killing villagers or to steal their stuff just for the hell of it - I felt encouraged to hold up my part in the role-playing" [51]). However, they also had expectations around VR increasing control in some aspects of the game, which it did not (e.g., "waving around 


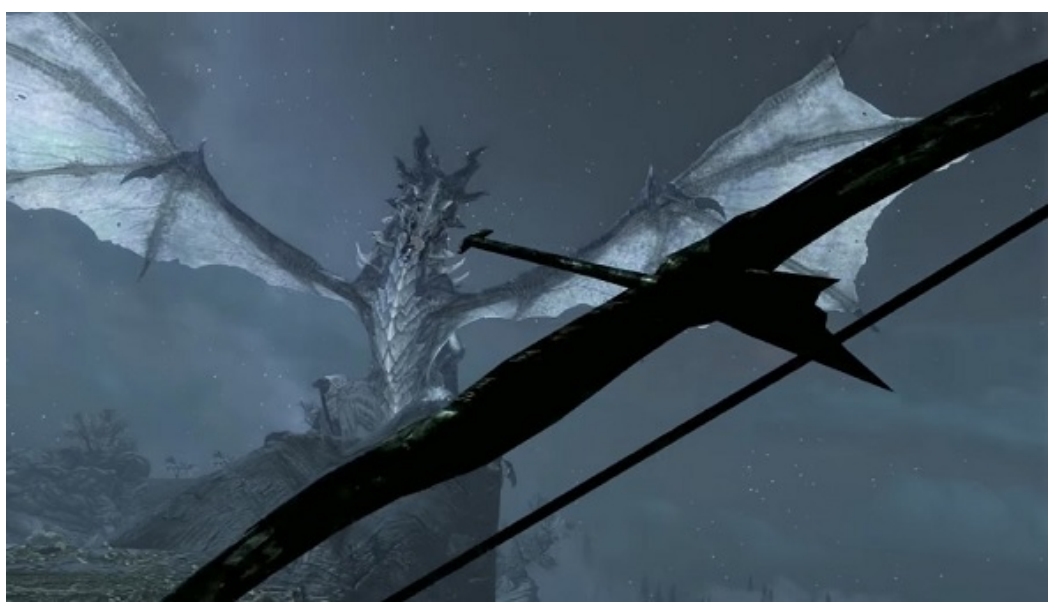

Fig. 2. Archery in Skyrim was not perceived to translate well to VR.

the sword feels like a bad Wii game and the bow and arrow barely work half the time" [32]). VR controls were also mentioned to be inaccurate at times, causing frustration (e.g., "when I have to fiddle with the controls or the menus and the whole concept falls apart" [19], "a constant inconvenience is the awkwardness of the controls" [52], "Elder Scrolls' famously terrible combat amplified by imprecise movements from the Move controllers" [41]). Reviewers mentioned that the interface is not well adapted to PC [16], which carries over to VR.

In Superhot, Control is afforded by the engagements, the weapons, and the movement. The player feels control due to the time-slowing mechanic and Superhot VR further increases this feeling (e.g., "as long as you hold still, you have all the time in the world to decide how to handle the situation ... that feeling of control is empowering" [69]). However, reviewers noted that every motion moves time forward and tracking issues in VR can make the game feel unfair (e.g., "the game is constantly interpreting the slightest twitch as an intended action, or simply imagining movement that you swear you never took" [33]). Superhot VR also includes a physical game object as a level progression tool, which reviewers noted can be missed if the player is not facing the right direction (e.g., "I got stuck at the end of a level because I'd looked the wrong way when the direction for what to do next appeared" [69]).

\subsection{Clear Goals}

There were no notable differences in affordances for Clear Goals between the VR versus non-VR games. In Skyrim, the Clear Goals element is afforded by an introductory narrative sequence that sets the overriding narrative and goals for the game, as well as an extensive array of main and side quests throughout the game. Reviewers paid attention to the procedurally generated questions, which gave players a never-ending supply of tasks. Superhot does not provide any explicit overriding goals, although the premise of "you're here to kill the red guys" [13] is made clear. Superhot provides the player with clear colour coding of objects or short text prompts for immediate objectives.

\subsection{Feedback}

The key differences in affordances for Feedback between the VR and non-VR games related to the Feedback criteria:

- Players should receive immediate feedback on their actions. 
In Skyrim, Feedback is afforded by the quest log, health and stamina bars, sounds used to denote successful actions, and text that shows up when a quest is complete or when the player has levelled up. Reviewers mentioned that Skyrim $V R$ has satisfying feedback for performing actions such as blocking and archery (e.g., "blocking sword swings and arrows with a shield by physically holding it out in front of you will never not be fun" [66]), but that there is a lack of visceral feedback (e.g., "hitting enemies with melee weapons largely feels like a hit-check rather than a blade slicing into flesh" [51]). The placement of the status bar was also noted as an issue (e.g., "I often found myself straining to find my health bar while running away from an enemy to see how much damage I'd taken" [51]). In Superhot, feedback is afforded by sounds for actions, text pop-ups when a level is completed, and visuals. Reviewers noted that in Superhot $V R$ there is a noticeable disconnect between the action of grabbing the level progression sign and it shattering (e.g., "that 'next' button comes in the form of a pyramid which asks you to 'grab' it, and then it breaks when you do ... don't ask me to 'grab' something and then have it break into pieces" [17]).

\subsection{Immersion}

The key differences in affordances for Immersion between the VR and non-VR games related to the Immersion criteria:

- Players should become less self-aware and less worried about everyday life or self.

- Players should feel emotionally involved in the game.

- Players should feel viscerally involved in the game.

In Skyrim, Immersion is afforded through the character interactions, the interactions with the environment, the story, and the sound design. Reviewers mention that the Skyrim VR player perspective gives the player a deeper sense of involvement ("the actual experience of being in $360^{\circ}$ virtual fantasy world is pretty amazing" [41], "creatures looking big on screen is one thing; towering over you is another" [52], "all of the action unfolds through your eyes" [14]) and that deeper emotional attachment is formed while playing the game (e.g., "experiencing Skyrim as an actual inhabitant of the world is an experience like no other and truly makes you feel like an epic adventurer" [67]). Although Skyrim is mentioned to offer a sense of visceral involvement, Skyrim VR is noted to afford increased visceral involvement from "intimate up close" interactions [70].

In Superhot, Immersion is afforded through the interactions with the environment, the story, the sound design, and the fast-paced action. Reviewers noted that Superhot $V R$ creates an experience where the player is so focused on the game that the rules are expected to transition over to real life (e.g., "I honestly found myself, ever briefly after taking off the headset, feeling like the real world should be slowing down around me as I moved more slowly" [17]). The VR version is mentioned to put the player into the shoes of an action hero who uses their own instincts to overcome challenge and to react to stimuli (e.g., "never in VR have I felt like the sort of action hero that Superhot VR has made me" [17], "by tapping into our natural survival instincts, it feels intuitive, if feels natural" [24]). Although the story is removed, the involvement remains high. Visceral involvement is noted to be higher in the VR version, with very real physical reactions (e.g., "very-real quantities of adrenaline still pumping through your veins and the pouring sweat from your brow" [24], "when I finally took off the headset, my hair was matted in sweat and my pulse was pounding" [17]).

\subsection{Comfort}

Comfort was a theme that emerged from the coding of the professional game reviews that was not covered by the existing GameFlow elements. The concept of Comfort was only present in VR game reviews. Comfort can be described 
as how comfortable a player feels while playing a VR game, with respect to physical issues such as play space, body position and movement, and cyber sickness. Comfort is often used as a subheading in professional reviews of VR games.

Skyrim VR affords Comfort by offering different control options to make the experience comfortable to players (e.g., "an option to disable 'realistic shield grip'" [51], "the game's locomotion system, offering both teleporting and free locomotion (controller relative), along with snap-turning and a fair set of options to adjust things as needed" [51]). Reviewers mentioned that some controller options could be nauseating to the players (e.g., "moving around freely is likely to cause at least a degree of nausea in most people" [32], "going without the snap [turning] option resulted in sickness after about an hour" [70]), while alternatives offered a less immersive experience (e.g., "while this can break the immersion, it does help newer users who haven't gained their VR legs acclimatise to free locomotion" [67], "if you can stomach it, I highly recommend smooth movement" [52]). It was noted that although the game does not eliminate the possibility of being nauseated, it does attempt to accommodate it. However, it was observed that nausea was likely to be an issue for most players in Skyrim VR, "due to the amount of time needed for grinding and exploration" [70]. The length of the game was mentioned as a general barrier to Skyrim as a comfortable VR game (e.g., "while I'm happy to while away the hours playing immense games like this, doing so with headset strapped to your face becomes needlessly tiring" [66], "Skyrim was made to be played sitting down for long stretches of time, which is just not that comfortable in VR" [19]). Reviewers also mentioned that Skyrim VR could be played sitting or standing, which is important given the length of the game (e.g., "I was able to play seated when I needed a break - the game automatically calibrates to a standing height, even when you're seated" [51]).

Superhot VR was mentioned to successfully confine its whole experience to a limited play area (e.g, "the game effectively keeps you facing forward toward the cameras to ensure you don't lose tracking" [17], "a standing game that usually doesn't require you to move your feet more than a step in any direction" [53]). To support the player in staying in place, defeated enemies eject their weapons for the player to grab, meaning that the player never has to move (e.g., "the fact that a dying enemy will effectively throw his gun at you ... is a very smart way to keep you from having to move too far" [53]). Although, some reviewers mentioned that having a large play space was preferable (e.g., "the need to dodge is going to take you a step or two in either direction, and you'll be stretching your arms out in desperate reach of weapons flying through the air" [17]).

\section{DISCUSSION}

Our research aimed to investigate enjoyment in VR games by analysing professional reviews of the VR and non-VR versions of two popular games, Skyrim and Superhot, using the GameFlow model as a theoretical framework. We found differences in how the elements of Feedback, Control, Immersion, and Player Skills were afforded in the VR and non-VR versions of the games.

We found that VR affords more control and easier interaction with a more direct connection between physical and virtual movements and actions. However, the more natural interfacing brings certain player expectations about how the game should feel and respond. The disconnect between actions and expected feedback can be jarring for the player. For example, archery in Skyrim VR and throwing in Superhot VR did not meet player expectations. Moreover, players had difficulty using these mechanics due to needing additional feedback to accurately perform the actions. Our results suggest that players need higher veracity feedback in VR games to meet expectations and to feel effective in their actions.

We also found that VR games can afford increased emotional and visceral immersion. Players can physically perform the actions that their character performs, bringing them into the experience. The act of physically performing 
Table 6. Proposed new GameFlow element of Comfort and associated criteria.

\begin{tabular}{ll}
\hline Comfort: & Games should afford players to feel physically comfortable while playing. \\
& Games should afford different players to control their level of comfort. \\
& Games should afford players to feel comfortable in their play space. \\
& Games should afford players to feel comfortable with their body position \\
& throughout play. \\
& Games should afford players to feel comfortable with their movements. \\
Games should afford players to minimise feelings of nausea.
\end{tabular}

these actions is fun to learn and provides satisfaction when performed correctly. Feelings of mastery at successfully performing actions, particularly if they are extraordinary or super-human, are amplified in VR. The player is no longer controlling a character to perform these actions, they feel as though they are doing it themselves. Emotional investment comes from the perspective of being the character and seeing through their eyes and the sheer impact of experiencing the world at-scale (e.g., life size dragons in Skyrim VR). This perspective and scale gives players a greater sense of presence and connection to the world, resulting in a greater impetus to act their part and an increased sense of consequence and responsibility for their actions.

We found that increased immersion often comes at the cost of a loss of sense of control for players. Reviewers felt limited in their abilities to freely move around both VR games, which impacted exploration in the open-world game Skyrim VR, but also reduced overall sense of control and continuity in Superhot VR. Reviewers were also critical of perceived inaccuracies in movement detection, particularly when it resulted in game outcomes that felt unfair (e.g., unintended movement in Superhot VR). Compromises were also made in player control as a result of limited input mechanisms (e.g., players choosing between turning during combat or two-handed wielding in Skyrim VR). The VR controllers or the control mappings were often found to not sufficiently accommodate the desired or required actions.

Comfort was identified to be a key enabler or inhibitor of player enjoyment in VR games. We propose that Comfort could be added to GameFlow as an additional element when designing or evaluating VR games. Comfort can present a direct barrier to playing, or becoming involved in, a VR experience, with a stand-out example being cyber sickness. There are also considerations related to size of the play space and how it is used, whether the player must stand for the duration of the game, and how they move their body. We defined Comfort as how comfortable a player feels while playing a VR game, with respect to physical issues such as play space, body position and movement, and cyber sickness. It is important to consider and support differences between players. Providing players with some level of control over their experience, even if it is choosing between immersion and comfort, is important to accommodate the needs of different players. We propose an additional GameFlow element of Comfort, as defined in Table 6, for use with XR and other games that blend the physical and virtual worlds.

A final point of interest that was identified in the professional reviews was that VR games, even complete and extensive games like Skyrim VR, can feel "more like a tech demo than a full experience” [17]. This perception warrants further investigation to fully understand, but it could relate to the experience feeling incomplete (e.g., lacking feedback) rather than the game being incomplete. Most reviews treated the VR games as an expansion or extension of the original games and limited their reviews to only the differences. Figure 3 illustrates the difference in focus between the reviews for Skyrim and Skyrim VR, in the form of word clouds for each set of reviews. The Skyrim reviews focused on the gameplay and narrative, whereas the Skyrim VR reviews focused more on the control system. 
One limitation of this work is that the VR games we analysed in this paper are both translations of existing PC games to VR. Our previous work has noted that Superhot can be seen as a successful translation of an existing game to VR [63]. However, some of the design decisions made in this translation could have had a small impact on the overall player experience, in terms of removing some in-game feedback, story context, and reducing the overall sense of control. Interesting future work lies in considering a broader set of VR games, including games that have been designed specifically for VR, such as Valve's recent Half-Life: Alyx.
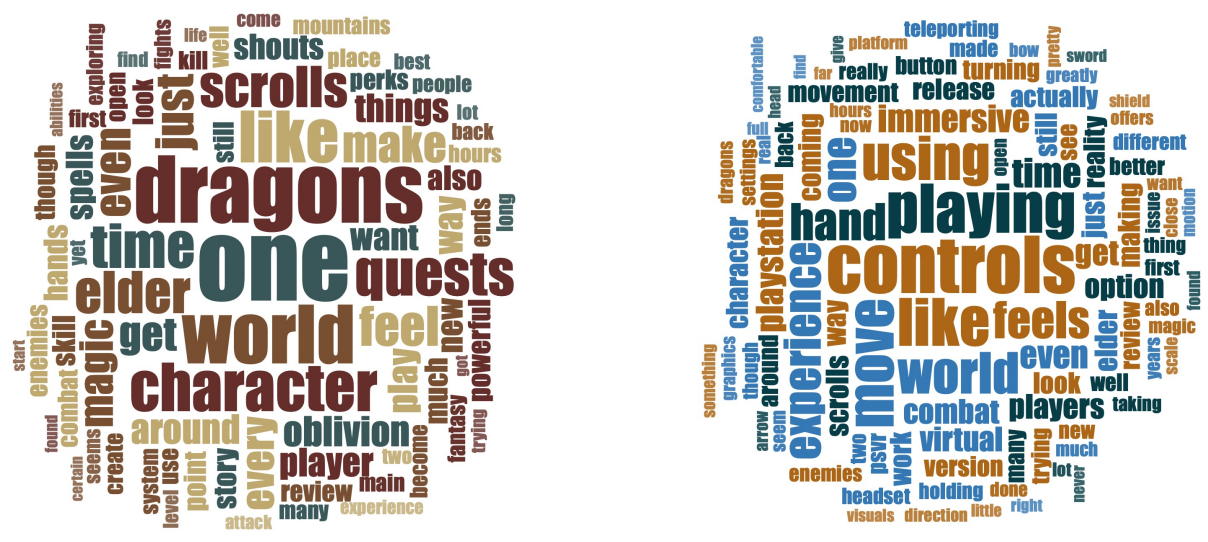

Fig. 3. Word clouds for Skyrim (top) and Skyrim VR reviews illustrate the difference in focus between the two sets of reviews.

\section{CONCLUSIONS}

We found that the existing elements of the GameFlow model are still applicable for the VR experience, with the element of social interaction not being relevant for the games analysed in this paper. Immersion, Feedback, Player Skills, and Control had more points of differentiation for the VR experience and helped to define a successful implementation of a VR game experience. Comfort exists between the physical and virtual spaces and represents a successful merging between the two. Comfort is necessary for an enjoyable gaming experience to occur. We conclude that many affordances of player enjoyment are consistent between VR and non-VR games, with VR both affording and inhibiting additional elements of enjoyment related to physical involvement and perception, expressed through immersion, feedback, control, and player skills and mastery. Player expectation is central to an enjoyable game experience and as we approach a more realistic simulation of the real world, particularly in how the player perceives and interacts in it, the more we see an uncanny valley type effect. The more lifelike the player's perceptions and interactions, the more the player expects from the simulation, including veracity of feedback and the subtleties of actions. Taking the player closer to a lifelike experience is a double-edged sword, in that past a certain threshold, anything short of perfection might not be enough.

\section{REFERENCES}

[1] Antonio Rafael Trigo Algar. 2014. Serious Games for Overcoming Phobias: The Benefits of Game Elements. Master's thesis. University of Skövde, Skövde, Sweden.

[2] Ferran Argelaguet Sanz, Franck Multon, and Anatole Lécuyer. 2015. A Methodology for Introducing Competitive Anxiety and Pressure in VR Sports Training. Frontiers in Robotics and AI 2 (2015), 10. https://doi.org/10.3389/frobt.2015.00010 
[3] Soumya C. Barathi, Michael Proulx, Eamonn O’Neill, and Christof Lutteroth. 2020. Affect Recognition Using Psychophysiological Correlates in High Intensity VR Exergaming. In Proceedings of the 2020 CHI Conference on Human Factors in Computing Systems (Honolulu, HI, USA) (CHI '20). ACM, New York, NY, USA, 1-15. https://doi.org/10.1145/3313831.3376596

[4] John Bedford. 2011. The Elder Scrolls V: Skyrim Review: Mine. Craft. Retrieved April 15, 2020 from https://www.eurogamer.net/articles/2011-11-10the-elder-scrolls-5-skyrim-review

[5] Alice Bell. 2016. Skyrim is fantasy that almost isn't fantasy, and that's why it's great. Retrieved April 15, 2020 from https://www.videogamer.com/ features/skyrim-is-fantasy-that-almost-isnt-fantasy-and-thats-why-its-great

[6] Matthew Bond and Russell Beale. 2009. What Makes a Good Game? Using Reviews to Inform Design. In Proceedings of the 23rd British HCI Group Annual Conference on People and Computers: Celebrating People and Technology (Cambridge, United Kingdom) (BCS-HCI '09). BCS Learning Development Ltd., Swindon, GBR, 418-422.

[7] Charles Boucher. 2011. The Elder Scrolls V: Skyrim Review. Retrieved April 15, 2020 from https://www.gamechronicles.com/reviews/pc/elderscrolls5/ skyrim.htm

[8] Peter Brown. 2016. Superhot Review: Time Lord. Retrieved April 15, 2020 from https://www.gamespot.com/reviews/superhot-review/1900-6416368/

[9] Sean Cahill. 2017. SuperHot VR Reviewd. Retrieved April 15, 2020 from https://www.gamingnexus.com/Article/5399/Superhot-VR/

[10] Eduardo H. Calvillo-Gámez, Paul Cairns, and Anna L. Cox. 2015. Assessing the Core Elements of the Gaming Experience. Springer International Publishing, Cham, 37-62. https://doi.org/10.1007/978-3-319-15985-0_3

[11] Victoria Clarke, Virginia Braun, and Nikki Hayfield. 2015. Thematic analysis. Qualitative psychology: A practical guide to research methods (2015), $222-248$.

[12] Mihaly Csikszentmihalyi. 1991. Flow: The Psychology of Optimal Experience. Harper Perennial, New York.

[13] Ben Davis. 2016. Review: Superhot. Retrieved April 15, 2020 from https://www.destructoid.com/review-superhot-343524.phtml

[14] Chris Dunlap. 2017. The Elder Scrolls V: Skyrim VR Review for PSVR. Retrieved April 15, 2020 from http://www.gaming-age.com/2017/11/elderscrolls-v-skyrim-vr-review-psvr/

[15] Samantha Finkelstein, Andrea Nickel, Zachary Lipps, Tiffany Barnes, Zachary Wartell, and Evan A. Suma. 2011. Astrojumper: Motivating Exercise with an Immersive Virtual Reality Exergame. Presence: Teleoperators and Virtual Environments 20, 1 (2011), 78-92. https://doi.org/10.1162/pres_a_00036

[16] Tom Francis. 2011. The Elder Scrolls V: Skyrim Review. Retrieved April 15, 2020 from https://www.pcgamer.com/au/the-elder-scrolls-v-skyrim-review/

[17] Javy Gwaltney. 2016. SuperHot VR: A Glimmer of Potential. Retrieved April 15, 2020 from https://www.gameinformer.com/games/superhot_vr/b/ oculusrift/archive/2016/12/12/a-glimmer-of-potential.aspx

[18] Kyle Hanson. 2016. Superhot Review. Retrieved April 15, 2020 from https://attackofthefanboy.com/reviews/superhot-review/

[19] Kyle Hanson. 2017. Skyrim VR Review. Retrieved April 15, 2020 from https://attackofthefanboy.com/reviews/skyrim-vr-review/

[20] Kyle Hanson. 2017. SuperHot VR Review. Retrieved April 15, 2020 from https://attackofthefanboy.com/reviews/superhot-vr-review/

[21] Kyle Hilliard. 2017. The Elder Scrolls V: Skyrim VR: The Worst Way To Experience Skyrim. Retrieved April 15, 2020 from https://www.gameinformer.com/games/the_elder_scrolls_v_skyrim_vr/b/playstationvr/archive/2017/11/18/the-elder-scrolls-v-skyrim-vrplaystation-vr-game-informer-review.aspx

[22] Meng-Hsuan Huang and Saiau-Yue Tsau. 2018. A Flow Experience Analysis on the Virtual Reality Artwork: La Camera Insabbiata. In Proceedings of the International Conference on Machine Vision and Applications (Singapore, Singapore) (ICMVA 2018). ACM, New York, NY, USA, 51-55. https://doi.org/10.1145/3220511.3220514

[23] Jeanne D. Johnston, Anne P. Massey, and Rickie Lee Marker-Hoffman. 2012. Using an Alternate Reality Game to Increase Physical Activity and Decrease Obesity Risk of College Students. Fournal of Diabetes Science and Technology 6, 4 (2012), 828-838. https://doi.org/10.1177/193229681200600414 PMID: 22920809.

[24] John-Paul Jones. 2017. SuperHot VR Review. Retrieved April 15, 2020 from https://www.psu.com/reviews/superhot-vr-review/

[25] Eng Tat Khoo, Adrian David Cheok, Ta Huynh Duy Nguyen, and Zhigeng Pan. 2008. Age invaders: social and physical inter-generational mixed reality family entertainment. Virtual Reality 12,1 (2008), 3-16. https://doi.org/10.1007/s10055-008-0083-0

[26] A.J. Maciejewski. 2017. SuperHot VR Review: Take the red pill. Retrieved April 15, 2020 from https://videochums.com/review/superhot-vr

[27] Anna Lisa Martin-Niedecken and Ulrich Götz. 2017. Go with the Dual Flow: Evaluating the Psychophysiological Adaptive Fitness Game Environment "Plunder Planet". In Serious Games, Mariano Alcañiz, Stefan Göbel, Minhua Ma, Manuel Fradinho Oliveira, Jannicke Baalsrud Hauge, and Tim Marsh (Eds.). Springer International Publishing, Cham, 32-43.

[28] Anna Lisa Martin-Niedecken and Elisa Mekler. 2018. The ExerCube : participatory design of an immersive fitness game environment. Serious Games : 4th foint International Conference, FCSG 2018, Darmstadt, Germany, November 7-8, 2018, Proceedings (2018), S.263-275. https://doi.org/10.1007/9783-030-02762-9_28

[29] Wytske Meekes and E. Stanmore. 2017. Motivational Determinants of Exergame Participation for Older People in Assisted Living Facilities: Mixed-Methods Study. Journal of Medical Internet Research 19 (2017).

[30] Metacritic. 2020. Metacritic. Retrieved April 15, 2020 from https://www.metacritic.com/

[31] Metro. 2016. SuperHot Review: cool ideal. Retrieved April 15, 2020 from https://metro.co.uk/2016/03/02/superhot-review-cool-idea-5729489/

[32] Metro. 2017. The Elder Scrolls V: Skyrim VR review: fantasy reality. Retrieved April 15, 2020 from https://metro.co.uk/2017/11/21/the-elder-scrolls-vskyrim-vr-review-fantasy-reality-7095099/

[33] Metro. 2017. SuperHot VR Review: cold reality. Retrieved April 15, 2020 from https://metro.co.uk/2017/07/25/superhot-vr-review-cold-reality-6805051/ 
[34] Logan Moore. 2017. SuperHot VR Review: Live. Die. Repeat. Retrieved April 15, 2020 from https://www.dualshockers.com/superhot-vr-review-psvrnew/

[35] Ann Morrison, Alessandro Mulloni, Saija Lemmelä, Antti Oulasvirta, Giulio Jacucci, Peter Peltonen, Dieter Schmalstieg, and Holger Regenbrecht. 2011. Collaborative use of mobile augmented reality with paper maps. Computers Graphics 35, 4 (2011), 789 - 799. https://doi.org/10.1016/j.cag.2011.04.009 Semantic 3D Media and Content.

[36] Mike Nelson and Scott Sharkey. 2011. The Elder Scrolls V: Skyrim PC Review. Retrieved April 15, 2020 from http://pc.gamespy.com/pc/elder-scrollsv/1212121p1.html

[37] Charles Onyett. 2012. The Elder Scrolls V: Skyrim Review - Say goodbye to real life. Retrieved April 15, 2020 from https://au.ign.com/articles/2011/11/ 10/the-elder-scrolls-v-skyrim-review

[38] Alanah Pearce. 2016. Superhot Review: A shooter where it pays to stop and think for a moment. Retrieved April 15, 2020 from https://au.ign.com/ articles/2016/02/25/superhot-review

[39] Cong Peng, Dangxiao Wang, Yuru Zhang, and Jing Xiao. 2019. A Visuo-Haptic Attention Training Game With Dynamic Adjustment of Difficulty. IEEE Access 7 (2019), 68878-68891. https://doi.org/10.1109/ACCESS.2019.2918846

[40] Dendi Permadi and Ahmad Rafi. 2016. Empirical Analysis of Mobile Augmented Reality Games for Engaging Users' Experience. In Intelligent and Evolutionary Systems, Kittichai Lavangnananda, Somnuk Phon-Amnuaisuk, Worrawat Engchuan, and Jonathan H. Chan (Eds.). Springer International Publishing, Cham, 343-355.

[41] Brendan Quinn. 2017. The Elder Scrolls V: Skyrim VR Review: Fresh Ideas, Bad Controls. Retrieved April 15, 2020 from https://www.cgmagonline.com/ reviews/elder-scrolls-v-skyrim-vr-review-fresh-ideas-bad-controls/

[42] Nimesha Ranasinghe, Koon Chuan Raymond Koh, Nguyen Thi Ngoc Tram, Yan Liangkun, Kala Shamaiah, Siew Geuk Choo, David Tolley, Shienny Karwita, Barry Chew, Daniel Chua, and Ellen Yi-Luen Do. 2019. Tainted: An olfaction-enhanced game narrative for smelling virtual ghosts. International fournal of Human-Computer Studies 125 (2019), 7 - 18. https://doi.org/10.1016/j.ijhcs.2018.11.011

[43] Kyle Rector, Roger Vilardaga, Leo Lansky, Kellie Lu, Cynthia L. Bennett, Richard E. Ladner, and Julie A. Kientz. 2017. Design and Real-World Evaluation of Eyes-Free Yoga: An Exergame for Blind and Low-Vision Exercise. ACM Trans. Access. Comput. 9, 4, Article 12 (April 2017), 25 pages. https://doi.org/10.1145/3022729

[44] Ben Reeves. 2016. Superhot Review: Slow But Sure. Retrieved April 15, 2020 from https://www.gameinformer.com/games/superhot/b/pc/archive/ 2016/02/26/superhot-game-informer-review.aspx

[45] Andrew Reiner. 2011. Skyrim Review: An RPG Worth Shouting About. Retrieved April 15, 2020 from https://www.gameinformer.com/games/the elder_scrolls_v_skyrim/b/pc/archive/2011/11/20/an-rpg-worth-shouting-about.aspx

[46] Mitchell Saltzman. 2016. Superhot Review: Bite Sized Brilliance. Retrieved April 15, 2020 from https://v1.escapistmagazine.com/articles/view/videogames/editorials/reviews/16773-Superhot-Review

[47] Phil Savage. 2016. Superhot Review. Retrieved April 15, 2020 from https://www.pcgamer.com/au/superhot-review-2/

[48] Jason Schreier. 2011. Review: Boundless Skyrim Will Become Your Life. Retrieved April 15, 2020 from https://www.wired.com/2011/11/skyrim-review/

[49] Brad Shoemaker. 2011. The Elder Scrolls V: Skyrim Review. Retrieved April 15, 2020 from https://www.giantbomb.com/reviews/the-elder-scrolls-vskyrim-review/1900-443/

[50] SSVAR. 2016. SuperHot VR Review. Retrieved April 15, 2020 from https://ssvar.ch/superhot-vr-review/

[51] SSVAR. 2017. Skyrim VR Review: The Other Side of the Immersion Equation. Retrieved April 15, 2020 from https://ssvar.ch/skyrim-vr-review-theother-side-of-the-immersion-equation/

[52] Dan Stapleton. 2017. The Elder Scrolls V: Skyrim VR Review. Retrieved April 15, 2020 from https://au.ign.com/articles/2017/11/22/the-elder-scrolls-vskyrim-vr-review

[53] Dan Stapleton. 2017. SuperHot VR Review. Retrieved April 15, 2020 from https://au.ign.com/articles/2017/08/04/superhot-vr-review

[54] Jim Sterling. 2011. Review: The Elder Scrolls V: Skyrim. Retrieved April 15, 2020 from https://www.destructoid.com/review-the-elder-scrolls-vskyrim-215507.phtml

[55] Mike Suskie. 2016. Superhot Review: It’s Pretty Attractive, Yeah. Retrieved April 15, 2020 from https://gamecritics.com/mike-suskie/superhot-review/

[56] Penny Sweetser. 2020. GameFlow 2020: 15 Years of a Model of Player Enjoyment. In Proceedings of the 32nd Australian Conference on HumanComputer-Interaction (Sydney, NSW, Australia) (OZCHI'20). ACM, New York, NY, USA.

[57] Penny Sweetser and Daniel Johnson. 2019. Evaluating the GameFlow Model with Different Stakeholders. In Extended Abstracts of the Annual Symposium on Computer-Human Interaction in Play Companion Extended Abstracts (Barcelona, Spain) (CHI PLAY '19 Extended Abstracts). ACM, New York, NY, USA, 697-703. https://doi.org/10.1145/3341215.3356286

[58] Penny Sweetser and Daniel Johnson. 2019. GameFlow and Player Experience Measures: An Initial Comparison of Conceptual Constructs. In Proceedings of the 31st Australian Conference on Human-Computer-Interaction (Fremantle, WA, Australia) (OZCHI'19). ACM, New York, NY, USA, 317-321. https://doi.org/10.1145/3369457.3369486

[59] Penny Sweetser, Daniel Johnson, and Jay Kyburz. 2020. Evaluating GameFlow in a Multiplayer Online Strategy Game Under Development. In Proceedings of the Australasian Computer Science Week Multiconference (Melbourne, VIC, Australia) (ACSW'20). ACM, New York, NY, USA, Article 47, 10 pages. https://doi.org/10.1145/3373017.3373068

[60] Penelope Sweetser, Daniel Johnson, and Peta Wyeth. 2013. Revisiting the GameFlow Model with Detailed Heuristics. The fournal of Creative Technologies 3 (May 2013). https://ojs.aut.ac.nz/journal-of-creative-technologies/index.php/JCT/article/view/16 
[61] Penelope Sweetser, Daniel Johnson, Peta Wyeth, Aiman Anwar, Yan Meng, and Anne Ozdowska. 2017. GameFlow in Different Game Genres and Platforms. Comput. Entertain. 15, 3, Article 1 (April 2017), 24 pages. https://doi.org/10.1145/3034780

[62] Penelope Sweetser, Daniel Johnson, Peta Wyeth, and Anne Ozdowska. 2012. GameFlow Heuristics for Designing and Evaluating Real-Time Strategy Games. In Proceedings of The 8th Australasian Conference on Interactive Entertainment: Playing the System (Auckland, New Zealand) (IE '12). ACM, New York, NY, USA, Article 1, 10 pages. https://doi.org/10.1145/2336727.2336728

[63] Penny Sweetser, Zane Rogalewicz, and Qingyang Li. 2019. Understanding Enjoyment in VR Games with GameFlow. In 25th ACM Symposium on Virtual Reality Software and Technology (Parramatta, NSW, Australia) (VRST '19). ACM, New York, NY, USA, Article 96, 2 pages. https: //doi.org/10.1145/3359996.3364800

[64] Penelope Sweetser and Peta Wyeth. 2005. GameFlow: A Model for Evaluating Player Enjoyment in Games. Comput. Entertain. 3 , 3 (July 2005), 3. https://doi.org/10.1145/1077246.1077253

[65] Roger Thomas Kok Chuen Tan, Vladimir Todorovic, James Keng Soon Teh, Goran Andrejin, Lee Shang Ping, and Adrian David Cheok. 2006. Metazoa Ludens. In ACM SIGGRAPH 2006 Sketches (Boston, Massachusetts) (SIGGRAPH '06). ACM, New York, NY, USA, 139-es. https://doi.org/10.1145/ 1179849.1180023

[66] Geoffrey Tim. 2017. Skyrim VR review: Fus VRo Dah. Retrieved April 15, 2020 from https://www.criticalhit.net/review/skyrim-vr/

[67] Sean Warhurst. 2017. Skyrim PSVR Review. Retrieved April 15, 2020 from http://www.impulsegamer.com/skyrim-psvr-review/

[68] Ryan Wedoff, Lindsay Ball, Amelia Wang, Yi Xuan Khoo, Lauren Lieberman, and Kyle Rector. 2019. Virtual Showdown: An Accessible Virtual Reality Game with Scaffolds for Youth with Visual Impairments. In Proceedings of the 2019 CHI Conference on Human Factors in Computing Systems (Glasgow, Scotland Uk) (CHI '19). ACM, New York, NY, USA, Article 141, 15 pages. https://doi.org/10.1145/3290605.3300371

[69] Daniel Weissenberger. 2017. SuperHot VR: The Gun Is Hardware. Retrieved April 15, 2020 from https://gamecritics.com/daniel-weissenberger/superhot$\mathrm{vr} /$

[70] Cory Wells. 2017. Review: The ElderScrolls V: Skyrim VR. Retrieved April 15, 2020 from https://www.hardcoregamer.com/2017/11/21/review-theelder-scrolls-v-skyrim-vr/280166/

[71] Matt Whittaker. 2016. Review: Superhot. Retrieved April 15, 2020 from https://www.hardcoregamer.com/2016/02/25/review-superhot/194642/

[72] Emilia Wittmers. 2012. Probabilistic Physical Games: Game Mechanics for Quantum Games. Master's thesis. Hasso Plattner Institute, Potsdam, Germany.

[73] Nynke Zwart. 2018. Assessing the Effect of Gamification on various aspects of Sport Exercises through an Augmented Reality Exertion Interface (Bachelor's Thesis). Technical Report. Radboud Universit, Nijmegen, Netherlands. 